\title{
Self-heating effect induced by ion bombardment on polycrystalline Al surface nanostructures evolution
}

\author{
H WANG*, Y ZHEN, H W JIANG and J T LIU ${ }^{\dagger}$ \\ Beijing Key Laboratory for Nano-Photonics and Nano-Structure, Department of Physics, Capital Normal University, \\ Beijing 100048, China \\ ${ }^{\dagger}$ Department of Physics, Nanchang University, Nanchang 330031, China
}

MS received 28 April 2011; revised 4 September 2011

\begin{abstract}
We studied the self-heating effect during ion bombardment process on polycrystalline Al foils. An anisotropic surface morphology evolution has been observed. The adjacent peaks' fusion along the direction perpendicular to the ion beam projection smoothen the surface. Fusion along the parallel direction has been suppressed due to $\mathrm{Ar}^{+}$ion bombardment. It attributes to the result of the competition between the isotropic thermal effect, due to the self-heating effect by energy exchange between incident ions and Al surface, and the suppression by continuous ion bombardment with a certain incident angle. Varying the incident ion beam angle with the angular range $32^{\circ}<\theta<82^{\circ}$, the ripple wave vector, $\lambda$, is found to be parallel to the ion beam direction, whereas for $\theta>82^{\circ}, \lambda$ is perpendicular to the beam direction. The critical angle, $\theta_{c}$, is close to $82^{\circ}$, which is different from Bradley and Harper's prediction and attributes to the self-heating effect.
\end{abstract}

Keywords. Ion bombardment; self-heating effect; morphology.

\section{Introduction}

Low energy ion beam sputtering (IBS) is one of the promising techniques for spontaneous nanopattern formation, such as ripples and dots, on the surface of the eroded materials (Facsko et al 1999; Mishra and Ghose 2006, 2008, 2009; Mollick and Ghose 2009; Krasheninnikov and Nordlund 2010). The phenomenon of ripples and nanodots formation has attracted much interest both for fundamental research and for the potential application in the field of nanotechnology. The first widely accepted theory describing the process of ripple formation was proposed by Bradley and Harper (1988). They predicted that the periodic nanostructure developed by IBS is the result of the interplay between the curvature-dependent sputtering and various surface relaxation processes. The surface curvature-dependent sputtering yields can be described by $-\left|v_{x}\right| \frac{\partial^{2} h}{\partial x^{2}}$ and $-\left|v_{y}\right| \frac{\partial^{2} h}{\partial y^{2}}$, here $v_{x}$ and $v_{y}$ are the sputtering rate along $x$ and $y$ direction in the sample plane, respectively $h$ the height of the sample surface in a localized area, which is a function of $x, y$ and the sputtering time, $t$. The surface diffusion can be described by $K \nabla^{2} \nabla^{2} h$ term, in which $K$ is a constant that depends on the surface diffusion process. If the surface diffusion is thermally activated, the coefficient, $K$, is given by $\frac{2 D \gamma \sigma}{n^{2} k_{\mathrm{B}} T}$, where $D$ is the surface self-diffusivity, $\gamma$ the surface free energy per unit area, and $\sigma$ the areal density of diffusing atoms. However, the smoothing term $K \nabla^{2} \nabla^{2} h$ is correct only for the

\footnotetext{
*Author for correspondence (wanghai@ mail.cnu.edu.cn)
}

isotropic surface diffusion. The anisotropic atoms diffusion on the surface of single crystal metal cannot be described by this term. On the surface of single crystal metal, the diffusion rate along different lattice indexes is different and is described by a $D \frac{\partial^{4} h}{\partial \vec{n}^{4}}$ term in which $\vec{n}$ represents the direction of the lattice index. Furthermore, the step-edge barrier due to the adatom (or vacancy) mobility on a single crystal metal surface, as is called Ehrlich-Schwoebel (ES) barrier, strengthens the anisotropic diffusion. The latter one can be summarized as $S \frac{\partial^{2} h}{\partial \vec{n}^{2}}$, where $S$ represents the ES diffusivity (Politi and Yillain 1996; Rusponi et al 1998; Costantini et al 2001). Apparently, if the energy scale of incident ions is much larger than the energy terms related with single crystal metal surface, BH model is applicable. However, in low energy ion beam range, a significant ES contribution term should be included (Fan et al 2005). In all, two basic crucial factors for ion bombardment surface nanostructures can be summarized as one is related to incident ion beam parameters such as incident energy, incident angle and ion flux; the other is the diffusion mechanism and process on the surface of materials.

It is noted that the energy exchange between incident ions and surface leads to a temperature rise, which modifies the diffusion behaviour locally at low ion energy and low ion flux. However, most previous experimental work has focused on the nanostructure formation under a fixed and uniform surface temperature by controlling the temperature of sample holder. Thus the thermal effect caused by the incident ion beam has been neglected. The purpose of the present work was to study this effect. In our experiment, the samples are 
thermally isolated from the sample holder in order to study the self-heating effect. Meanwhile, a polycrystalline Al foil is selected to rule out ES barrier or anisotropic diffusion term on the surface. We call the thermal effect induced by incident ion bombardment as self-heating effect. It is observed that the surface morphology is strongly influenced by the selfheating effect induced by ion bombardment.

\section{Experimental}

Al foils with $0.1 \mathrm{~mm}$ thickness were used. The Al sample was degreased with acetone first, then washed in de-ionized water. After this cleaning process, the aluminum was electropolished in a mixture of perchloric acid and ethanol (1:4 in volume) under d.c. voltage of $10 \mathrm{~V}$ for $3 \mathrm{~min}$ at $6^{\circ} \mathrm{C}$. The samples were washed again in de-ionized water and dried before being mounted into vacuum chamber. The base pressure of the ion sputtering system was better than 2.5 $\times 10^{-4} \mathrm{~Pa}$. During ion sputtering, it rose to $1.8 \times 10^{-2} \mathrm{~Pa}$ due to the back-filling of argon with a purity of $99.999 \%$. The temperature of the water-cooled substrate holder was fixed at $15^{\circ} \mathrm{C}$. The $\mathrm{Ar}^{+}$energy was kept at $600 \mathrm{eV}$ and the ion flux was fixed with $2.5 \times 10^{-13} \mathrm{ions} / \mathrm{cm}^{2} / \mathrm{s}$, which was much lower compared with the most previous work. The angle of ion incidence with respect to the surface normal, $\theta$, can vary in the range of $0^{\circ}-92^{\circ}$. Poly tetra fluoro ethylene (PTFE, thickness of $0.2 \mathrm{~mm}$ ) slices were used as thermal isolator between $\mathrm{Al}$ foil and the holder, a schematic diagram is inserted in figure 1. The temperature was measured by in situ thermometer during $\mathrm{Ar}^{+}$ion bombardment. The surface morphologies were examined by ex situ AFM and SEM.

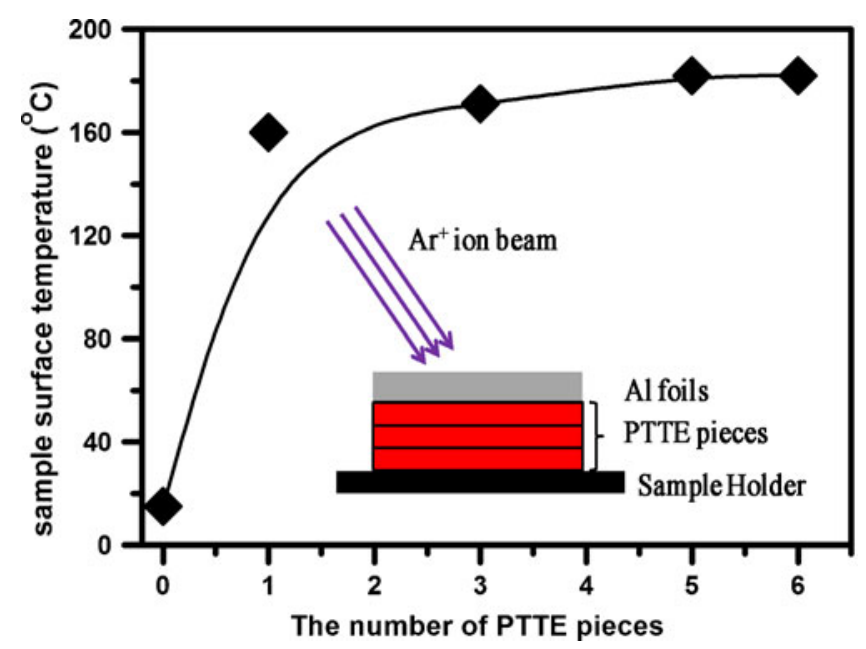

Figure 1. Surface temperature vs piece of PTFE films. Inset shows sketch map of experimental set up.

\section{Results and discussion}

The inserted PTFE pieces can effectively isolate the thermal loss through the sample holder. Figure 1 shows the surface temperature of $\mathrm{Al}$ foils vs number of pieces of PTFE slices. The surface temperature rises with increasing sputtering time and is stabilized after $30 \mathrm{~min}$ bombardment. The temperature of $\mathrm{Al}$ rises abruptly to $162^{\circ} \mathrm{C}$, when one piece of PTFE is inserted between $\mathrm{Al}$ foil and the sample holder. The temperature of Al foil's surface saturates while five slices of PTFE are used. It indicates that the $\mathrm{Al}$ foil is completely thermally isolated from the holder and the thermal balance constructed by the energy exchange between the incident ion and $\mathrm{Al}$ foil. Since the highest temperature is around $180^{\circ} \mathrm{C}$ under $30 \mathrm{~min}$ ion bombardment, obviously, the thermal radiation from $\mathrm{Al}$ foil can be neglected. It is speculated that this relation might be significantly enhanced in a high energy and long time ion bombardment process.

Figure 2 shows a sequence of the evolution of the surface topography of $\mathrm{Al}$ foils with increasing pieces of PTFE. The incident angle is fixed as $62^{\circ}$, which is within the critical angle range predicted by $\mathrm{BH}$ theory (Bradley and Harper 1988). It can be seen that needles develop on the surface of the sample attached on the well cooled substrate holder. Needles grow larger with rise of surface temperature and eventually fuse together. It is noted that the roughening dominates equally in both the ion beam direction and its vertical direction when ion beam incidence angle is within the critical angle range. However, in our experiment, the fusion is highly anisotropic on the surface. Fusion along the direction perpendicular to the ion beam projection smoothes the surface. Fusion along the parallel direction is suppressed due to $\mathrm{Ar}^{+}$ ion bombardment. The topography is the result of the competition between fusion of adjacent peaks and $\mathrm{Ar}^{+}$ion bombardment. It is noted that there is no anisotropic energy term, such as ES barriers, existing on the polycrystalline Al foils surface. However, the isotropic thermal diffusion leads to an anisotropic morphology evolution, which can be understood as the interaction between continuous ion bombardment and isotropic diffusion on the surface.

The morphology features can be explicated by the statistical data of the grain density and the surface root mean square (rms) roughness from figure 2, as shown in figures 3(a) and (b). In figure 3(a), the first data point (dash-circle) is for the sample fabricated in well water-cooled condition (without PTFE). The grain density increases when one piece of PTFE is used. Further increasing the pieces of PTFE leads to the rise of surface temperature, while the grain density decreases due to the interfusion between adjacent grains. However, when the sample is completely isolated, the grain density decreases significantly. As described by BH theory, the relationship between the surface rms roughness and the sample temperature is that the surface roughness drops monotonically with increasing sample temperature. Therefore, increase in the sample temperature always contributes to the surface smoothening, i.e. the higher the sample temperature is, the smoother the surface becomes 

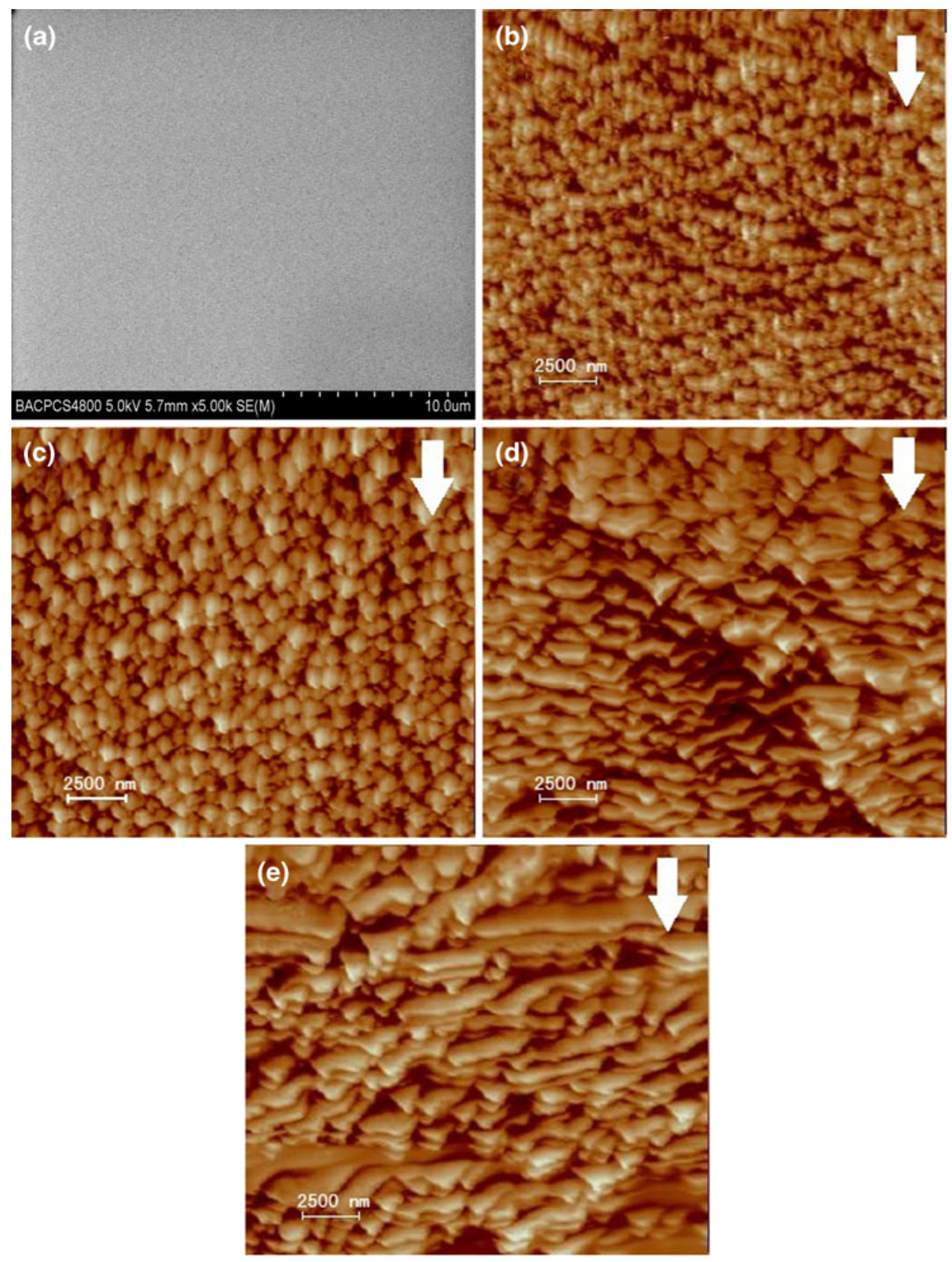

Figure 2. (a) SEM image of un-bombarded Al surface, (b)-(e) $20 \times 20 \mu \mathrm{m}^{2}$ AFM images of sputtered surfaces with different pieces of PTFE: (b) water-cooled, (c) one piece of PTFE, (d) three pieces of PTFE, (e) five pieces of PTFE. Sputtering time is 30 min. Projected ion beam direction is from top to bottom.

(Krasheninnikov and Nordlund 2010). On the other hand, figure 3(b) reveals that the roughness increases with temperature increasing from 15 to $165^{\circ} \mathrm{C}$. This might be due to the highly localized thermal effect at low energy range of ion bombardment. Ion bombardment dominates along the whole surface, and rise in temperature promotes the bombardment effect rather than smoothening the surface. Thus the surface nanostructure might be influenced by the thermal effect caused by ion bombardment and the eventual morphology might be different from the prediction of $\mathrm{BH}$ theory. Figure 3(b) also shows that the roughness declines at higher temperature range due to the adjacent structures fusion.

The influence of the self-heated effect induced by ion bombardment on the surface morphology depends on the erosion time. Figure 4 shows evolution of the surface topography vs sputtering time for the samples with five pieces of PETT. Thin sheet structures are formed after $10 \mathrm{~min}$ 
bombardment as shown in figure 4(a). With the sputtering time increasing up to $20 \mathrm{~min}$, thin sheets become contiguous due to the diffusion and fusion on the surface as shown in figure 4(b). The eventual morphology with $30 \mathrm{~min}$ bombardment has been shown in figure $2(\mathrm{~d})$. The time evolution in our samples is more complicated than the one with a stable sample surface temperature. In our experiments, the surface
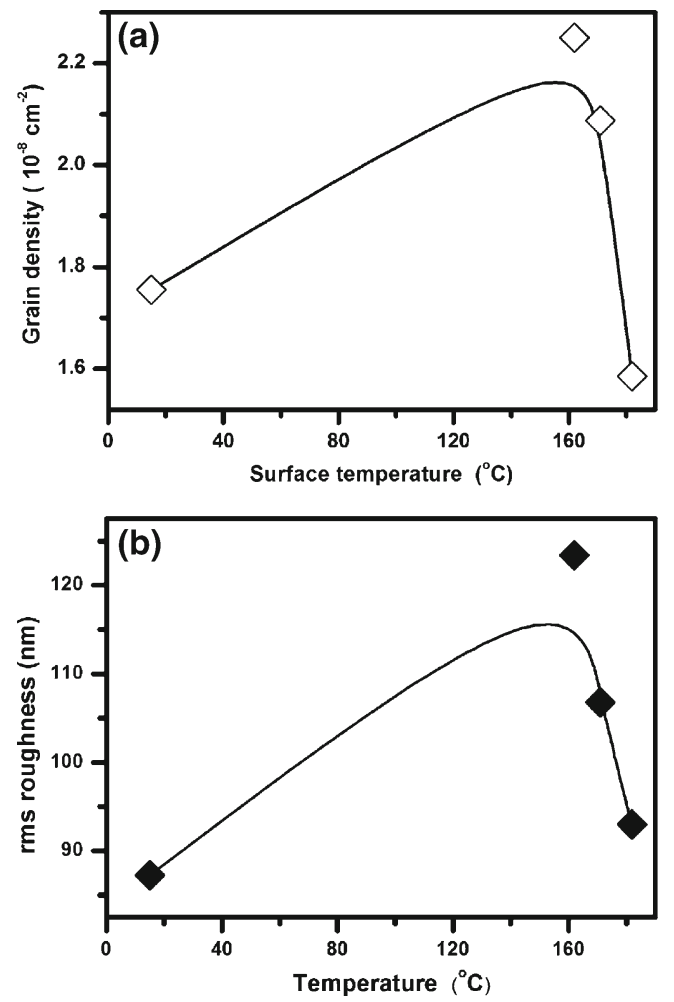

Figure 3. (a) Grain density vs sample surface temperature and (b) surface roughness vs sample surface temperature. diffusion strongly depends on the temperature rising process, which again confirms the importance of the self-heating effect on the nano-morphology formation. In the case of high energy and high flux bombardment, the thermal balance will be set up quickly.

Furthermore, based on the above results, it can be expected that the critical angle $\theta_{\mathrm{c}}$ predicted in $\mathrm{BH}$ theory varies due to the self-heating effect. Such expectation is also confirmed by our experiments as shown in figure 5 . The orientation of the ripples changes with the angle of ion incidence according to BH theory. As the incident angles is below $\theta_{\mathrm{c}}$, the ripple wave vectors are parallel to the ion beam projection (parallel mode ripples), whereas for angles above $\theta_{\mathrm{c}}$, the ripple wave vectors are perpendicular to the ion beam projection (vertical mode ripples). $\theta_{\mathrm{c}}$ is about $60^{\circ}$ as predicted in BH theory (Bradley and Harper 1988) and confirmed by the experiments (Mishra and Ghose 2006, 2008, 2009). Figure 5 shows some representative SEM images of the sputtered Al surfaces at different incident angles, when the sample is thermally isolated. It can be seen that while $\theta<82^{\circ}$, the wave vector of ripples is parallel to the ion beam direction, whereas for $\theta>82^{\circ}$, ripples with perpendicular wave vectors to the beam direction are formed. In the case of $82^{\circ}$, both parallel and perpendicular features can be seen on the surface. Thus the critical angle, $\theta_{\mathrm{c}}$, is close to $82^{\circ}$, which is quite different with the observations of Bradley and Harper (1988). The change of the critical angle is due to the impact of the self-heating effect.

\section{Conclusions}

In summary, a self-heating effect induced by ion bombardment on the polycrystalline Al surface's morphology evolution is clearly evidenced. At a fixed incident angle, ripples are formed when the sample surface reaches a certain
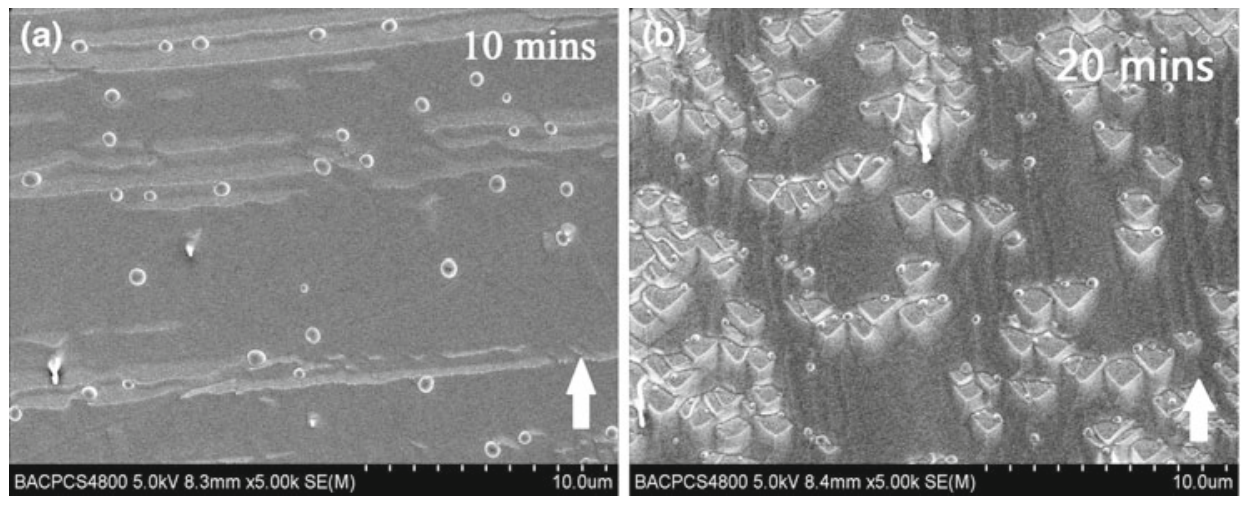

Figure 4. Evolution of surface topography vs sputtering time when sample was thermally isolated. Sputtering time is (a) $10 \mathrm{~min}$, (b) $20 \mathrm{~min}$. Projected ion beam direction is from bottom to top. 

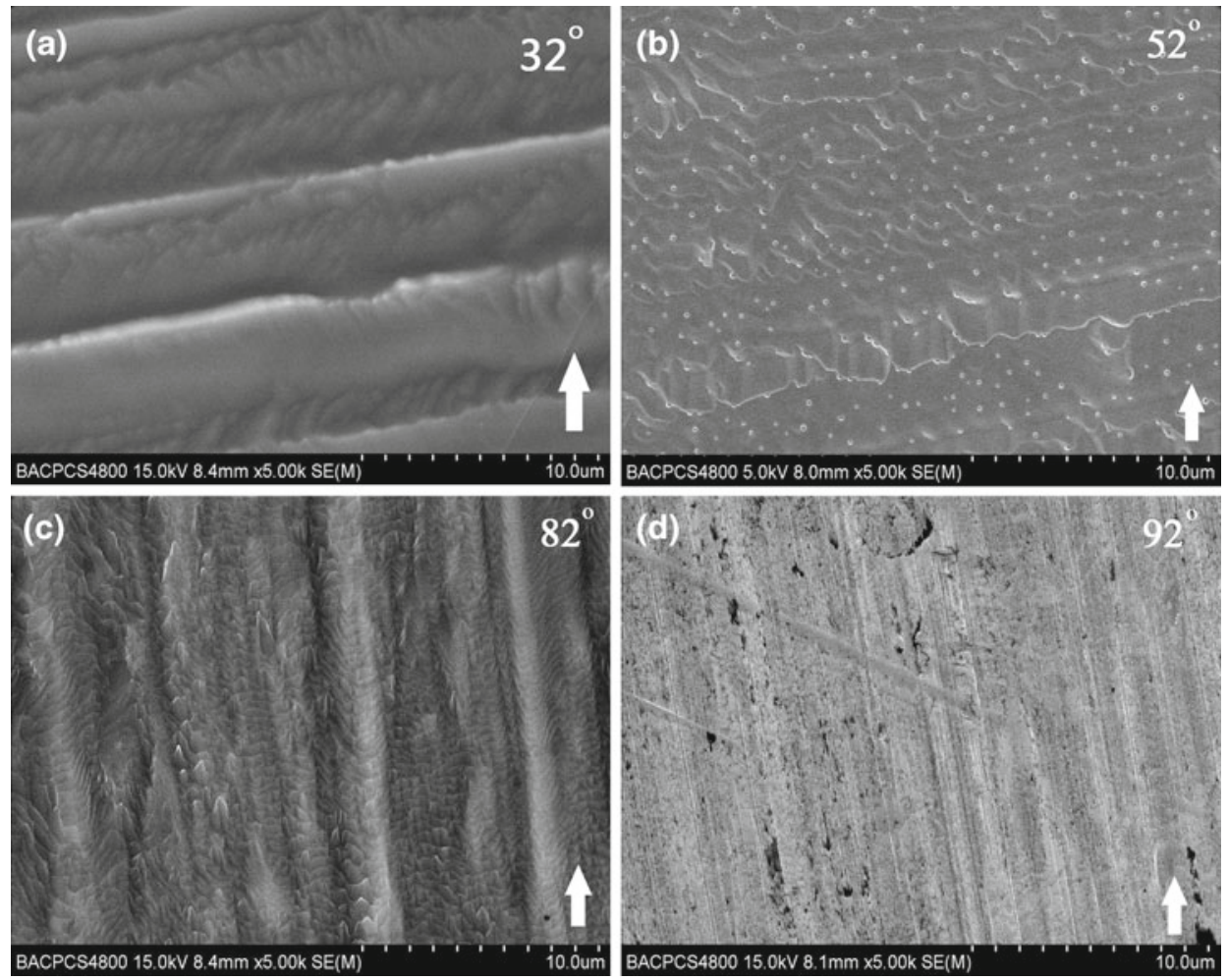

Figure 5. (a)-(d) are evolution of surface topography vs incident angle when sample was thermally isolated. Sputtering time is $30 \mathrm{~min}$ and incident angles are indicated in each image. Projected ion beam direction is from bottom to top.

temperature caused by the self-heating effect. The critical angle for the ripple rotation is $82^{\circ}$, which is quite different from the BH theory's prediction. It indicates that at low ion energy and low ion flux regime, the self-heating effect induced by ion bombardment cannot be neglected. The dynamic process of the self-heating effect vs sputtering time, angles and ion energy needs to be further explored, respectively. The studies of the self-heating effect on single crystals are expected to understand the surface diffusion process under continuous ion bombardment in detail.

\section{Acknowledgements}

This work was supported by the NSFC Grant Nos. 10904097 and 10904059, PHR(IHLB) from Beijing and the NSF from Jiangxi Province (Nos. 2008GZW0003 and 2009GQW0017).

\section{References}

Bradley R M and Harper J M E 1988 J. Vac. Sci. Technol. A6 2390 Costantini G, Rusponi S, de Mongeot F B, Boragno C and Valhusa U 2001 J. Phys.: Condens. Matter 135875

Facsko S, Dekorsy T, Koerdt C, Trappe C, Kurz H, Vogt A and Hartnaget H L 1999 Science 2851551

Fan Wen-bin, Li Wei-qing, Qi Le-jun, Sun Hai-tong, Luo Jia, Zhao You-yuan and Lu Ming 2005 Nanotechnology 161526

Krasheninnikov A V and Nordlund K 2010 J. Appl. Phys. 107 071301

Mishra P and Ghose D 2006 Phys. Rev. B74 155427

Mishra P and Ghose D 2008 J. Appl. Phys. 104094305

Mishra P and Ghose D 2009 J. Appl. Phys. 105014304 (and references therein)

Mollick S A and Ghose D 2009 J. Appl. Phys. 106044309

Politi P and Yillain J 1996 Phys. Rev. B54 5114

Rusponi S, Costantini G, Boragno C and Valbusa U 1998 Phys. Rev. Lett. 812735 